\title{
Measuring Contact Points from Displacements with a Compliant, Articulated Robot Hand
}

\author{
Gurdayal S. Koonjul, Garth J. Zeglin, and Nancy S. Pollard \\ The Robotics Institute, Carnegie Mellon University, Pittsburgh, PA, 15213, USA
}

\begin{abstract}
Manipulators with compliant actuation exhibit passive joint displacements when exposed to external forces or collisions. This paper demonstrates that this displacement information is sufficient to infer a coarse estimate of the location of an incidental collision. Three techniques for contact point detection are compared: a closed-form inference model based on a serial chain with joint springs, a variation on Self Posture Changeability, and an empirical memory-based model of joint trajectories. The methods were experimentally tested using a Shadow Hand on an industrial Motoman SDA10 arm to quantify localization performance, actively discover and avoid a thin obstacle and localize and grasp a cup.
\end{abstract}

\section{INTRODUCTION}

The addition of force and tactile sensors to multi-fingered robot manipulators adds cost, bulk and design constraints. We are interested in detecting collision using only joint angle measurements on compliant multi-fingered manipulators while performing dexterous tasks in changing or unknown environments.

Collision contact information can be used as control feedback when performing object detection or manipulation tasks. In this paper we compare theoretical and empirical methods to interpret joint angle data to perform obstacle avoidance. The output of each method provides a location for a single collision along the gripper. We demonstrated these methods with a Shadow Hand gripper attached to a Motoman SDA10 dual-arm robot. We also performed a simple experiment to demonstrate object localization.

The first of the three methods uses a simple closed form analytical model of a serial-chain finger with compliant joint actuation subjected to a single disturbance force. This approximates the palm and a single finger of the Shadow Hand, which has fingers that are tendon-driven by compliant actuators. We measured a virtual spring stiffness at each joint experimentally and constructed a stiffness function which we solve for the disturbance force and location given the joint angles.

The second method uses a variation on Kaneko and Tanie's Self Posture Changeability (SPC), a method in which a manipulator with one compliant joint estimates

Manuscript received September 15, 2010.

G. S. Koonjul is with The Robotics Institute, Carnegie Mellon University, 5000 Forbes Avenue, Pittsburgh, PA 15213, USA (phone: 412330-9355, email: gkoonjul@andrew.cmu.edu).

G. J. Zeglin is with The Robotics Institute, Carnegie Mellon University (email: garthz@cmu.edu).

N. S. Pollard is with The Robotics Institute, Carnegie Mellon University (email: nsp@cs.cmu.edu). contact point location by imposing a small posture change with other actuated joints while maintaining contact. The intersection of the manipulator surface over the two postures indicates the point of contact. We extend the idea to accommodate multiple compliant joints.

The third method consists of building a classifier from joint angle displacement data to model a single collision during an axial motion of the hand. We set the Shadow Hand into an exploratory posture and collided with an obstacle placed at different points across the path of motion. We observed that the joint trajectories during collision are linearly separable and we used a nearest neighbor method to classify a new test trajectory to the means of a set of training trajectories at each contact point.

We used a test set of 500 collisions with each method and found that our empirical model performed with higher precision and recall than the other methods. We demonstrate application of this method for obstacle avoidance and object localization in the accompanying supplement video. Using the empirical model, the Shadow Hand is shown to localize and navigate around an obstacle. Subsequently, the Shadow Hand is shown to grasp a cup after localizing the rim.

\section{RELATED WORK}

Several researchers have addressed the estimation of manipulator contact state from purely kinematic data, but the area seems underexplored.

Deckers, Dollar and Howe[1] describe a formulation of contact estimation as a POMDP model. The theoretical model is presented without simulation or hardware validation. Like our work, the model estimates a discrete contact state from the displacement of compliant joints using an active motion process, but unlike our approach uses a probabilistic foundation.

Haidacher and Hirzinger[2] present a solution for a multifingered hand with compliance-controlled joints to infer contacts from joint angle data by actively exploring poses within the vicinity of a stable grasp and analyzing multiple pose measurements as kinematic constraints on contact location. They tested the solution on a simulation of a DLR Hand II. The motion generates measurements of multiple nearby stable grasps; these constraints on the contact locations are combined using either an optimization or an observer.

Kaneko and Tanie[3] demonstrate a method for estimating a single contact location using "self-posture changeability" (SPC) by actively controlling the motion and compliance of a single finger and treating the link poses as kinematic 
constraints on a contact. The method is tested in hardware on a three-DOF finger. After contact is initially detected heuristically, one joint is controlled to be compliant and another to move along a prescribed trajectory. The compliant joint allows the link in contact to slip or roll at the contact point such that the location of contact can be inferred from the intersection of multiple measurements of the surface plane. As implemented, the compliance control utilizes torque sensors, but the method would only require joint angle sensing if one joint included physical compliance.

Huber and Grupen[4] build upon the SPC ideas to allow estimation of contact location during normal task motions. The algorithm uses observers which use joint angles to compute kinematic constraints that estimate feasible contacts, then joint torques to disambiguate the kinematic result and estimate forces. The result is tested both in simulation and on a MIT-Utah hand.

Siegel[5] analyzes the estimation of an equilibrium grasp state using only joint angle data and tests several methods in simulation and on a MIT-Utah hand. The work assumes the grasped object belongs to a small set of objects with known geometry, which implies the set of feasible grasps is finite. Two classifiers are presented which only require joint angles to identify the grasp, the first using an automatically generated decision tree and the second a memory-based nearest-neighbor method.

A number of authors including [6][7] have addressed sensing contact location using internal force and torque measurements. These methods use structural strain sensors to estimate external forces and are thus simpler than distributing many active tactile sensors over the surface.

However, we are interested in even simpler solutions which take advantage of joint compliance. Our methods avoid the complexity of adding strain gages or torque sensors and use only the existing joint sensors to determine contact location.

\section{HAND SETUP}

The Shadow Hand is mounted to one of the 7-DOF robot arms of a Motoman SDA10. The Shadow Hand System is a manipulator resembling the human hand with 24 degrees of freedom of which 20 are actuated. The distal joints of the fingers (excluding thumb) are coupled to the medial joints to approximate human tendon constraints. The finger flexion joints are actuated over $-20^{\circ}$ to $90^{\circ}$ degrees to a precision of $\pm 1^{\circ}$. Each joint is driven by a tendon pair attached to a total of 36 McKibben actuators and four return springs. The joints are position controlled but remain compliant due to the elasti-

Table 1: Shadow Hand Parameters

\begin{tabular}{cl}
\hline \hline Parameter & Length \\
\hline Palm length & $9.75 \mathrm{~cm}$ \\
MF3 link length & $4.52 \mathrm{~cm}$ \\
MF2 link length & $2.48 \mathrm{~cm}$ \\
MF1 link length & $3.00 \mathrm{~cm}$ \\
Shadow Hand forearm & \\
diameter (the cluster of & $7.62 \mathrm{~cm}$ \\
McKibben actuators) & \\
\hline \hline
\end{tabular}

city of the pneumatic artificial muscle actuators. The middle finger which we are using for contact has three links with joints labeled from tip to base (e.g. MF1 refers to the distal joint, MF2 the medial, etc.). While the Shadow Hand does have tactile sensors mounted on the finger and palm surfaces, we do not use their output at all in our experiments. Table 1 shows a select set of measurements of the physical structure of the Shadow Hand.

\section{TASK DESCRIPTION}

For our obstacle avoidance task we assume that all hand trajectories are axial motions, i.e. parallel to the Shadow Hand forearm. This assumption guarantees contact with an obstacle only occurs on the compliant fingers of the hand and not on the rigid robot arm. We would like to detect all collisions over the swept volume of the arm. This requires a hand pose which will incur some deflection of the compliant joints for any contact of interest. We call this posture the exploratory posture and the space over which a posture can detect obstacles the detectable space. Given that initial exploratory trajectories are aligned with the Shadow Hand forearm axis, this only requires response to collisions in front of the bounding cylinder depicted in Fig. 1. We further assume the obstacle is a vertical edge, which reduces the required detectable space to the band illustrated as a dark region on the cross section.

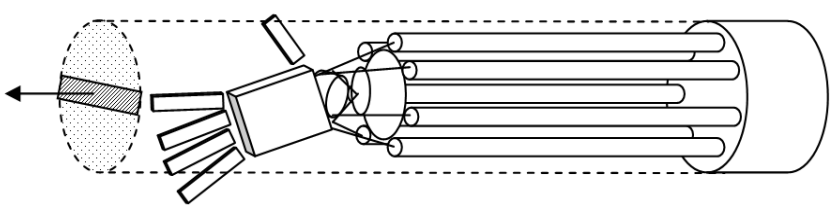

Fig. 1. Region of contact collision and Shadow Hand

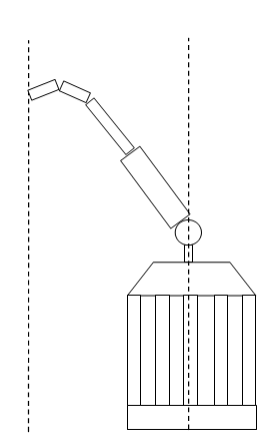

(a)

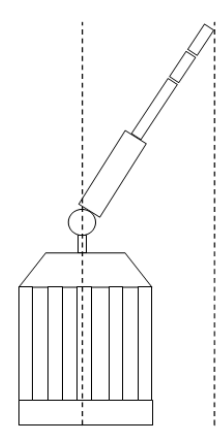

(b)

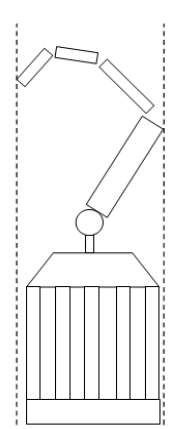

(c)
Fig. 2 (a), (b), (c). Possible Hand Postures and their detectable spaces

We can ensure both of these conditions by choosing a posture that responds to different points of collision uniquely and covers the entire width of the forearm. Fig. 2 shows three candidate posture choices, of which only Fig. 2(c) satisfies both these conditions. Any collision along the finger pose in Fig. 2(c) guarantees a response from at least one compliant joint, since each possible contact has a non-zero lever arm around at least one joint. As a counter example, in Fig. 2(a) and (b) collisions directly in line with the wrist joint would show no response.

We chose to use only the middle finger of the Shadow Hand for exploration in order to increase sensitivity. 
Additional fingers in contact increase total stiffness which reduces the observed deflection. The final parameters selected for the exploratory posture of the hand were MF1: 55 $5^{\circ}, \mathrm{MF} 2: 30^{\circ}, \mathrm{MF} 3: 70^{\circ}$ and wrist angle: $-33^{\circ}$. This choice was motivated by the observation that our Shadow Hand consistently drifts into this particular pose from nearby commanded poses (within $\pm 3^{\circ}$ ), possibly due to hysteresis, backlash and static friction. The other fingers of the hand are flexed to their flexion joint limits (i.e. $90^{\circ}$ at each joint).

The obstacle chosen for experimentation was a $1 \mathrm{~cm}$ radius smooth round rigid pole to emulate point contact collisions.

\section{Methods For Detecting Point Contact: Closed FORM MODEL}

\section{A. Assumptions}

In order to develop a closed form model of the Shadow Hand, we assume that the compliance of each finger joint can be represented by a linear torsional spring at each joint, with neutral angle set at the chosen exploratory posture. Because the hand is only actuated in the plane we neglect gravity and the small mass of the finger.

\section{B. Theory}

Consider the $m$-link serial chain with torsional springs shown at rest in Fig. 3(a) and the accompanying state after a force has been quasi-statically applied to the system in 3(b). Each link is named from base to tip as Link $1,2, \ldots, n$ with link lengths $l_{i}(i=1,2, \ldots, n)$. Each joint angle $\theta_{i}$ is defined relative to the previous link. The torsional springs at each joint have a spring stiffness of $k_{i}$. A force $\mathrm{F}$ is applied on the last link, at a distance $l_{f}$ from the last joint, displacing each joint angle by $\theta_{d i}$. Let $\bar{\theta}_{l}$ represent a joint angle relative to the base:

$$
\overline{\theta_{l}}=\theta_{1}+\theta_{2}+\cdots+\theta_{i}
$$

Because the system is in equilibrium, the torque due to the force is equivalent to the torque due to the torsional spring at each joint.

Where

$$
\tau_{\text {spring }}=\tau_{\text {force }}
$$

$$
\left[\begin{array}{c}
\tau_{1_{\text {spring }}} \\
\tau_{2_{\text {spring }}} \\
\vdots \\
\tau_{n_{\text {spring }}}
\end{array}\right]=\left[\begin{array}{c}
\tau_{1_{\text {force }}} \\
\tau_{2} \text { force } \\
\vdots \\
\tau_{n_{\text {force }}}
\end{array}\right]
$$

Let $\mathrm{R}$ represent the vector from the base joint to the location of the force on the last link. The torque due to the force about the base joint is:

$$
\tau_{1_{\text {force }}}=R \times F
$$

And is equal to the torque due to the torsional spring at joint 1:

$$
\tau_{1_{\text {spring }}}=k_{1} \overline{\theta_{d 1}}
$$

For the other joints we have to consider the reaction force at the base joint which is given by:

$$
B=-F
$$

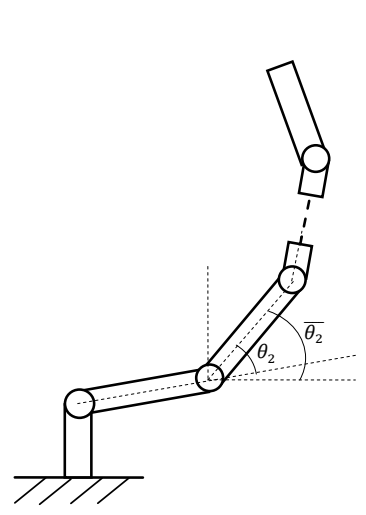

(a)

Fig. 3. (a) Serial chain at equilibrium due to springs (b) Quasi static equilibrium with an applied force

Each successive joint has an equilibrium equation:

Where

$$
k_{i} \overline{\theta_{d l}}=k_{i-1} \overline{\theta_{d(l-1)}}+B \times L_{d(i-1)}
$$

$$
L_{i}=\left\{\begin{array}{l}
l_{i} \sin \left(\bar{\theta}_{l}\right) \\
l_{i} \cos \left(\bar{\theta}_{l}\right)
\end{array}\right\}
$$

A stiffness matrix can be constructed such that the spring torques are given by:

Where

$$
\tau_{\text {spring }}=K Q
$$

$$
\begin{aligned}
& K= \\
& {\left[\begin{array}{ccccc}
k_{1}+k_{2} & -k_{2} & & & 0 \\
& \ddots & & & \\
& & -k_{i} & k_{i+1}+k_{i+2} & -k_{i+2} \\
& & & \\
0 & -k_{n-2} & k_{n-1}+k_{n} & -k_{n} \\
& & -k_{n} & k_{n}
\end{array}\right]} \\
& Q=\left[\begin{array}{c}
\overline{\theta_{d 1}} \\
\overline{\theta_{d 2}} \\
\vdots \\
\overline{\theta_{d n}}
\end{array}\right]
\end{aligned}
$$

Using equations 7, 8 and 9, we can find the corresponding elements of the force torque vector given in Equation 2. If the force acts on link $p$ then the elements of this torque vector are given by equations 10-12.

$$
\text { For } i=1,2, \ldots, p-1 \quad \begin{aligned}
\tau_{i_{\text {force }}} & =F \times L_{i} \\
\tau_{p_{\text {force }}} & =F \times L_{f}
\end{aligned}
$$

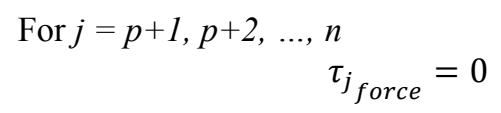

The elements of the $\tau_{\text {force }}$ vector are zero for joints beyond the contact point. The link in contact corresponds to the last non zero row of $\tau_{\text {force }}$. For example, if the force acted on the second joint, the $\tau_{\text {force }}$ would be as shown in 14 . 


$$
\tau_{\text {force }}=\left[\begin{array}{c}
F \times L_{1} \\
F \times L_{f} \\
0 \\
\vdots
\end{array}\right]
$$

In a given collision, $Q$ is measured and so $\tau_{\text {spring }}$ is easily computed using the physical parameters $k_{n}$. The only unknowns of the equilibrium equation (2) are $F$ (direction and magnitude) and $l_{f}$. At least three elements of $\tau_{\text {spring }}$ must be non zero in order to solve for all three unknowns. This corresponds only to collisions on the third link or higher. If we assume zero friction, then the force is normal to the link in contact, and that would constrain our equation to two unknowns and solve for collisions on the second link as well. Given the exploratory posture, contact can never be made to the palm, so we will always have at least two non zero elements.

\section{Stiffness Measurements}

To determine the virtual spring stiffnesses, the Shadow Hand was set to the exploratory posture, and an Imada force gauge was pressed perpendicularly at each link tip. Using equilibrium Equation 3, we calculated the stipulated torques $F \times L_{i}$ (using the force gauge readings) and then simultaneously solved for the spring constants using Equation 9. Ten experiments were done on each joint and the average virtual stiffnesses are given in Table 2. A comparison of the stipulated torques calculated from the force gauge readings and the calculated stiffnesses are shown in Fig. 4. The curves are of the appropriate magnitude and generally linear, so the assumption that the joint compliance can be modeled as a linear spring is reasonable. The diversion of the curves in the MF3 figure is due to the MF3 joint reaching a joint limit due to the high force applied.

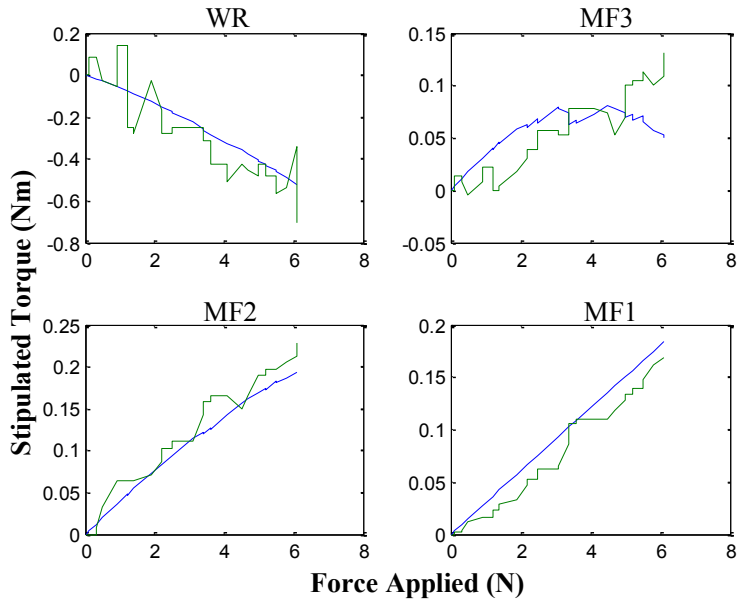

Fig.4. Stipulated Torques. Smooth blue curve calculated from force gauge readings, green curve from Equation 9 using virtual spring stiffnesses.

Table 2. Stiffness Results (in N-m/radians)

\begin{tabular}{lc}
$k_{1}($ wrist $)$ & $45.72 \pm 9.55$ \\
$k_{2}(m f 3)$ & $5.33 \pm 2.69$ \\
$k_{3}(m f 2)$ & $1.64 \pm 0.86$ \\
$k_{4}(m f 1)$ & $0.49 \pm 0.16$ \\
\hline
\end{tabular}

\section{Methods For Detecting Point Contact: SPC}

We augment Kaneko and Tanie's Self Posture Changeability method to use more than one compliant joint. The extra degrees of freedom imply that there may be more than one possible intersection of the SPC motions. However, we assume that an obstacle makes contact at only one link and remains in contact on that link after an SPC motion. This guarantees that one of the observed geometric intersection points is the correct collision point. Fig. 5 illustrates the concept and how the intersection of the finger posture after an SPC motion represents the contact position. The SPC motion we use is a forward linear motion increment up to a maximum of $1.2 \mathrm{~cm}$ (this is the maximum distance that can be travelled before MF3 reaches a joint limit given our chosen posture and any collision along the collision region). At each increment, we find the intersection point(s) between the initial posture and the current posture as the intersection point is indicative of the point of contact. If no intersection or multiple intersections spanning across multiple links are found then the collision is labeled as indeterminate. However, a result can still be found in the latter case if the link in contact is known (this would eliminate other intersection points and pinpoint the correct contact area). This concept is discussed in Section VIII.

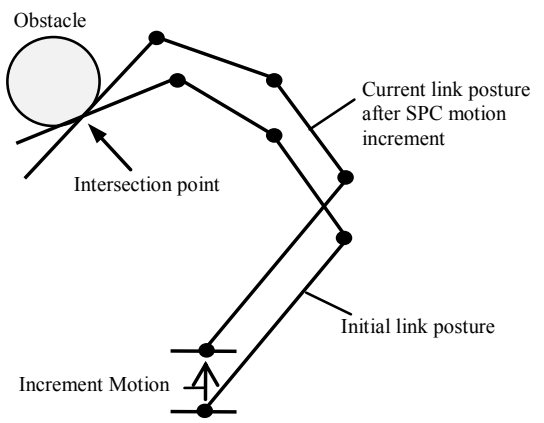

Fig.5. SPC motion from an initial to a final posture to detect contact location

\section{Methods For Detecting Point Contact: EMPIRICAL MODEL}

\section{A. Construction}

We constructed an empirical model that represents joint angle displacement data versus contact collision location. The Shadow Hand was set to the exploratory posture and made to collide with the obstacle at 10 contact locations, chosen over the surface of the middle finger, as depicted in Fig. 6. Joint angle state and robot arm position was recorded during the trials. Time of contact was measured when the joint angle displacement achieved a threshold of $2^{\circ}$. This value was chosen based on the variability and noise of joint values during typical position control of the Shadow Hand to hold a pose. After the onset of contact, the hand is moved $1.2 \mathrm{~cm}$ forward to create a deflection in the compliant joints. All joint angle displacement data during this sequence is considered collision data.

The goal of the model is to map the joint angle displacements to contact locations. The collision data comprises the Wrist, MF1, MF2 and MF3 joint angle displacements. Fig. 7 (a) shows a plot of collision data on 
each of the 10 contact locations in the three dimensional space of MF1, MF2, and MF3. Fig. 7(b) shows displacement data for 10 collisions on the first contact location.

Classifying a new collision event is performed by using a nearest neighbor method to find the closest class representing contact location. The mean of each class is precomputed from 20 collision training examples collected at each contact location. The method uses one of four distance metrics to compare a new test sample to the training means: Manhattan, Euclidean Squared, Mahalanobis and Cosine Similarity. They are briefly described below.

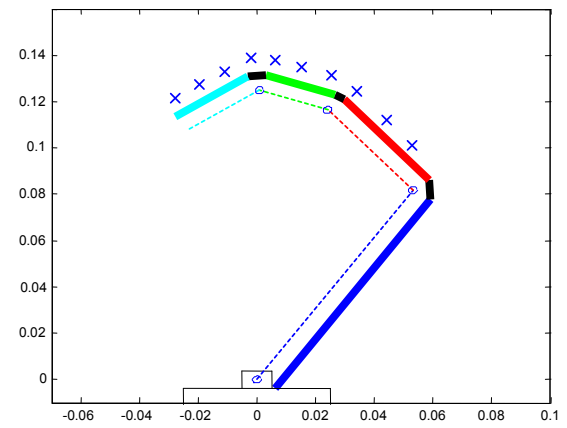

Fig. 6. Simulation showing contact locations and exploratory posture. Center line of the middle finger marked by dashed line. Bold line represents finger surface.

\section{B. Distance Metrics}

Four distance metrics are used to compare a test displacement set to the means of the displacement data at each contact location from a training set. For any test vector $x$ and mean of each collision set $\mu$, the distance between them measured by manhattan (MAN), euclidean squared (ES), mahalanobis (MAH), and cosine similarity(COS) are:

$$
\begin{gathered}
d_{M A N}(x, \mu)=|x-\mu| \\
d_{E S}(x, \mu)=|| x-\left.\mu\right|^{2} \\
d_{M A H}(x, \mu)=\sqrt{(x-\mu) \Sigma^{-1}(x-\mu)} \\
d_{C O S}(x, \mu)=\frac{x \cdot \mu}{\|\mu\| \cdot\|\mu\|}
\end{gathered}
$$

Where $\Sigma$ is the covariance all points in the collision set.

\section{TESTING AND MEthod SET UP}

In order to best compare the performance of all three methods we used a testing set of collision data from 50 collisions at each contact location. This test set was created using the same procedure explained in Section VII.

The closed form method requires the identity of the link in contact to determine which torque balance equations to use when solving for the contact location. Recall from Equation 15 that the link in contact simply corresponds to the first link that experiences a non-zero torque. In practice however, we often found that all the links experienced non-zero torques regardless of the collision location. We therefore provide the identity of the link to the method beforehand. Thus for each point in a test collision data trajectory, the closed form method can solve for the contact location using the correct force balance equations.
When using the SPC method, we found that the geometry of our finger configuration led to multiple intersection points, which made identifying the point of contact ambiguous. We therefore provide the identity of the link in contact to the SPC method as well and so each point in a test trajectory is compared to the reference posture until an intersection is found that lies on the provided link in contact. If no solution is found the trajectory is labeled indeterminate.

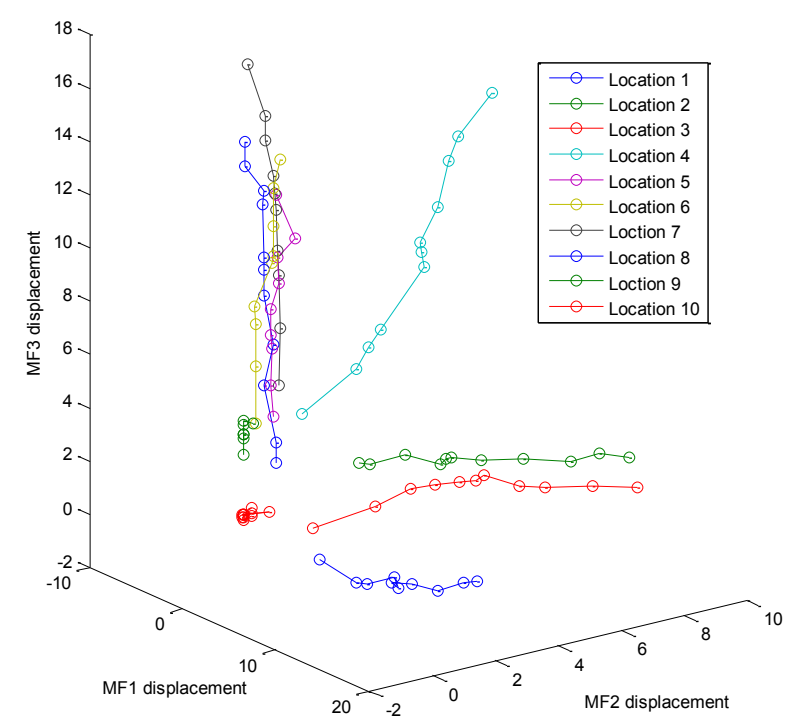

(a)

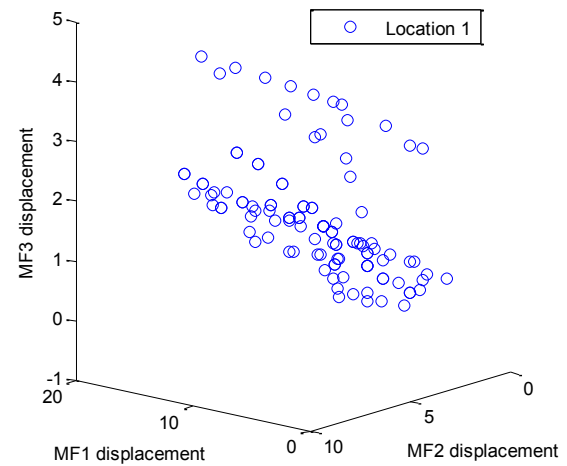

(b)

Fig. 7 (a) Plot of MF1, MF2 and MF3 displacement data for one collision at each contact location.

(b) Plot of MF1, MF2, and MF3 displacement data for ten collisions at contact location 1 .

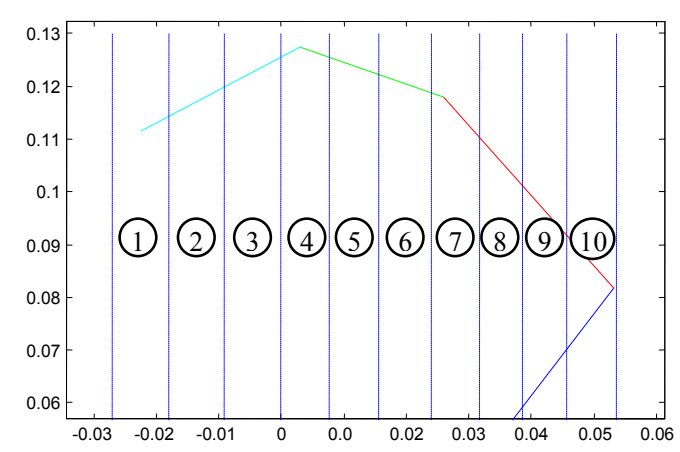

Fig. 8. Bin locations

The empirical model training set consists of collision data from 20 collisions at each contact location; collected using 
the same method described in Section VII. An eleventh "cluster" is included comprising non-collision displacement data. The empirical model uses the final point of a test collision trajectory for classification.

We also used the 10 possible contact locations to create regions or bins of contact location, shown in Fig. 8. We can then quantize the results of the closed form method and SPC method output into one of these bins and compare them to the results of the empirical method on the same test set.

\section{RESUlTS AND DISCUSSION}

Tables 3, 4, and 5 represent histograms of the results of submitting each test trial to each classification method. Each column represents a contact location tested, and each row represents a classifier output bin. The perfect classifier would thus be depicted as a matrix with values only in the diagonal. The additional row in each table represents cases that were indeterminate by the method. For the closed form model and SPC method, these indeterminate values include the test cases that were classified to a contact location inconsistent with the provided link in contact. These infeasible outputs are represented by the grayed out areas. The empirical model is not given the link in contact so all output bins are always feasible. Indeterminate cases in the empirical model results represent trajectories that were classified as non-contact.

Table 5 depicts the histogram of the results using the cosine similarity distance metric to classify samples; this metric was the best of the four tested. An LDA analysis was also performed to transform the training and testing data in an attempt to maximize cluster separation, but the results of the analysis did not show a significant gain that would justify its use.

It is important to note that in all methods, an indeterminate classification is not necessarily a system failure. Because the existence of contact can be trivially determined using thresholding, if the location cannot be estimated a control system can retry the collision after possibly performing a reset, dither or retreat to take a new reading and reattempt classification.

Table 3. Closed Form Method Results

\begin{tabular}{crrrrrrrrrr}
\hline \hline Actual Position & 1 & 2 & 3 & 4 & 5 & 6 & 7 & 8 & 9 & 10 \\
\hline Classified in & & & & & & & & & & \\
Bin 1 & $\mathbf{2 4}$ & 4 & 0 & 0 & & & & & & \\
Bin 2 & 13 & $\mathbf{3 8}$ & 32 & 1 & & & & & & \\
Bin 3 & 2 & 2 & $\mathbf{1 1}$ & 49 & & & & & & \\
Bin 4 & 1 & 0 & 1 & $\mathbf{0}$ & 2 & 0 & 0 & & & \\
Bin 5 & & & & & $\mathbf{2 1}$ & 2 & 0 & & & \\
Bin 6 & & & & & 19 & $\mathbf{2 0}$ & 41 & & & \\
Bin 7 & & & & & 0 & 7 & $\mathbf{2}$ & & & \\
Bin 8 & & & & & & & & $\mathbf{0}$ & 3 & 0 \\
Bin 9 & & & & & & & & 3 & $\mathbf{5}$ & 0 \\
Bin 10 & & & & & & & & 37 & 19 & $\mathbf{4 6}$ \\
\hline Indeterminate & 10 & 6 & 6 & 0 & 8 & 21 & 7 & 10 & 23 & 4 \\
\hline \hline
\end{tabular}

Table 4. SPC Method Results

\begin{tabular}{crrrrrrrrrr}
\hline \hline Actual & & & & & & & & & & \\
Position & 1 & 2 & 3 & 4 & 5 & 6 & 7 & 8 & 9 & 10 \\
\hline Classified in & & & & & & & & & & \\
Bin 1 & $\mathbf{9}$ & 4 & 5 & 3 & & & & & & \\
Bin 2 & 23 & $\mathbf{2 4}$ & 17 & 7 & & & & & & \\
Bin 3 & 15 & 22 & $\mathbf{2 7}$ & 14 & & & & & & \\
Bin 4 & 0 & 0 & 0 & $\mathbf{2 5}$ & 11 & 5 & 3 & & & \\
Bin 5 & & & & & $\mathbf{1 0}$ & 13 & 12 & & & \\
Bin 6 & & & & & 24 & $\mathbf{2 3}$ & 24 & & & \\
Bin 7 & & & & & 4 & 3 & $\mathbf{0}$ & & & \\
Bin 8 & & & & & & & & $\mathbf{2 9}$ & 22 & 19 \\
Bin 9 & & & & & & & & 16 & $\mathbf{9}$ & 7 \\
Bin 10 & & & & & & & & 4 & 19 & $\mathbf{2 3}$ \\
\hline Indeterminate & 3 & 0 & 1 & 1 & 1 & 6 & 11 & 1 & 0 & 1 \\
\hline \hline
\end{tabular}

Table 5. Empirical Model Results using Cosine Similarity

\begin{tabular}{|c|c|c|c|c|c|c|c|c|c|c|}
\hline $\begin{array}{c}\text { Actual } \\
\text { Position } \\
\end{array}$ & 1 & 2 & 3 & 4 & 5 & 6 & 7 & 8 & 9 & 10 \\
\hline \multicolumn{11}{|l|}{ Classified in } \\
\hline Bin 1 & 26 & 14 & 1 & 0 & 0 & 0 & 0 & 0 & 0 & 1 \\
\hline Bin 2 & 21 & 33 & 14 & 0 & 0 & 0 & 0 & 0 & 0 & 0 \\
\hline Bin 3 & 2 & 3 & 34 & 1 & 0 & 0 & 1 & 0 & 0 & 0 \\
\hline Bin 4 & 0 & 0 & 0 & 49 & 5 & 1 & 0 & 0 & 0 & 1 \\
\hline Bin 5 & 0 & 0 & 0 & 0 & 23 & 12 & 12 & 10 & 1 & 9 \\
\hline Bin 6 & 0 & 0 & 1 & 0 & 4 & 33 & 14 & 0 & 2 & 3 \\
\hline $\operatorname{Bin} 7$ & 0 & 0 & 0 & 0 & 18 & 3 & 11 & 18 & 0 & 1 \\
\hline $\operatorname{Bin} 8$ & 0 & 0 & 0 & 0 & 0 & 1 & 12 & 15 & 19 & 1 \\
\hline Bin 9 & 1 & 0 & 0 & 0 & 0 & 0 & 0 & 7 & 27 & 9 \\
\hline Bin 10 & 0 & 0 & 0 & 0 & 0 & 0 & 0 & 0 & 0 & 25 \\
\hline Indeterminate & 0 & 0 & 0 & 0 & 0 & 0 & 0 & 0 & 1 & 0 \\
\hline \multicolumn{11}{|c|}{ Table 6. Method Success Rates in Percent (\%) } \\
\hline Method & \multicolumn{2}{|c|}{$\begin{array}{c}\text { Exact Rate } \\
\text { (Average } \\
\text { Recall) }\end{array}$} & \multicolumn{2}{|c|}{$\begin{array}{l}\text { Average } \\
\text { Precision }\end{array}$} & \multicolumn{2}{|c|}{$\begin{array}{l}\text { Link } \\
\text { Rate }\end{array}$} & \multicolumn{2}{|c|}{$\begin{array}{c}\text { Left or } \\
\text { Right } \\
\text { Rate }\end{array}$} & \multicolumn{2}{|c|}{$\begin{array}{c}\text { Indeter- } \\
\text { minate } \\
\text { cases }\end{array}$} \\
\hline $\begin{array}{l}\text { Closed } \\
\text { Form }\end{array}$ & \multicolumn{2}{|c|}{33.40} & \multicolumn{2}{|c|}{39.43} & \multicolumn{2}{|c|}{80.11} & \multicolumn{2}{|c|}{82.33} & \multicolumn{2}{|c|}{19.00} \\
\hline $\mathrm{SPC}$ & \multicolumn{2}{|c|}{35.80} & \multicolumn{2}{|c|}{34.86} & \multicolumn{2}{|c|}{94.72} & \multicolumn{2}{|c|}{95.42} & \multicolumn{2}{|c|}{5.00} \\
\hline \multicolumn{11}{|c|}{ Empirical Model using: } \\
\hline Manhattan & \multicolumn{2}{|c|}{35.40} & \multicolumn{2}{|c|}{44.13} & \multicolumn{2}{|c|}{78.17} & \multicolumn{2}{|c|}{88.42} & \multicolumn{2}{|c|}{1.20} \\
\hline $\begin{array}{c}\text { Euclidean } \\
\text { Squared }\end{array}$ & \multicolumn{2}{|c|}{40.20} & \multicolumn{2}{|c|}{49.45} & \multicolumn{2}{|c|}{77.39} & \multicolumn{2}{|c|}{88.75} & \multicolumn{2}{|c|}{1.40} \\
\hline Mahalanobis & \multicolumn{2}{|c|}{51.40} & \multicolumn{2}{|c|}{67.30} & \multicolumn{2}{|c|}{74.33} & \multicolumn{2}{|c|}{84.50} & \multicolumn{2}{|c|}{0.34} \\
\hline $\begin{array}{c}\text { Cosine } \\
\text { Similarity }\end{array}$ & & & & 8.73 & & 67 & 97 & & $\mathbf{0 .}$ & \\
\hline
\end{tabular}

Table 6 shows classification success rates for each method at several categories of precision. The first column shows the average recall (true positives over the sum of true positives and false negatives) or the rate of classifying contact exactly into the correct bin. The second is average precision (true positives over the sum of true and false positives). The third is the success rate for identifying the link in contact, regardless of the exact contact position. This is more appropriate for analyzing the results of the empirical model. The fourth column is the success rate for classifying 
a test case to the left or right of the exploratory posture (bins 1 to 4 represent "right hand side" contact and bins 5 to 10 represent "left hand side" contact). Even this single bit is useful since we are intending to use these methods for obstacle avoidance. After a collision, the arm must circumnavigate the obstacle and so any method to determine the contact location should at the very least provide a coarse estimate of the position and allow the arm to clear the obstacle (by moving left or right). The final column lists the ratio of indeterminate classifications.

The closed form method showed poor results for collisions on the link close to the base, possibly due to the no-friction assumption required at that link. Additionally, the estimate of the virtual spring stiffnesses have high variance but could likely be improved by constructing an apparatus to characterize the displacement forces instead of using a handheld force gauge.

The high success rates for left or right classification using the empirical model should be expected. In Fig. 7 (a) we observe a large degree of separation from trajectories of collision on the right side of the finger and on the left. We should also note that the elliptical shape of the training trajectories should favor Mahalanobis distance and cosine similarity over the other distance metrics and this is reflected in the results.

\section{CONCLUSIONS AND Future WORK}

We explored three different methods for detecting single contact collision location on a serial link manipulator using only joint displacements. The first two methods require the identity of the link in contact with the obstacle in order to determine a contact location. While in this work we provided that information directly to the methods, this information could instead be potentially provided by additional sensors, a heuristic on the joint angles or by an empirical model that trained and classified by 'link in contact' instead of by contact location. We could also explore alternative increment motions, such as controlled movements of particular finger joints after a collision.

While the SPC method showed greater overall accuracy on all links than the closed form method, both methods were still inundated by unclassifiable collisions. In practice, these collisions would have to be reattempted in order to find a better result.
The empirical model method showed comparable results to the other two methods without prior information of the link in contact. The method could correctly classify the link in contact $88 \%$ of the time and give a correct coarse estimate of obstacle location to the left or right of the manipulator $98 \%$ of time. We successfully demonstrated the application of this method in the accompanying supplement video depicting obstacle avoidance and object localization to grasp a cup. Some stills are shown in Fig. 9.

All of the methods described use the passive compliance properties of a manipulator to generate coarse contact collision location using incidental joint angle displacement data. As demonstrated in the work, manipulation tasks using collision feedback can be performed using only kinematic data. The idea could be extended to different arm trajectories and hand postures, and other tasks such as surface following. Including multiple fingers would provide a three dimensional contact area that could be used to detect obstacle shape, height or perform rough three-dimensional object reconstruction.

\section{REFERENCES}

[1] P. Deckers, A. M. Dollar, and R. D. Howe, "Guiding grasping with proprioception and Markov models," in Proceedings of the Workshop on Robot Manipulation: Sensing and Adapting to the Real World, Robotics: Science and Systems Conference (RSS), (Atlanta, GA), 2007.

[2] S. Haidacher and G. Hirzinger, "Contact point identification in multifingered grasps exploiting kinematic constraints," in Proceedings of IEEE International Conference on Robotics and Automation, vol. 2, pp. $1597-1603,2002.2$

[3] M. Kaneko and K. Tanie, "Contact point detection for grasping an unknown object using self-posture changeability," IEEE Transactions on Robotics and Automation, vol. 10, pp. 355-367, June 1994

[4] M. Huber and R. A. Grupen, "2-D contact detection and localization using proprioceptive information," IEEE Transactions on Robotics and Automation, vol. 10, pp. 23-33, February 1994.

[5] D. M. Siegel, Pose Determination of a Grasped Object Using Limited Sensing. PhD thesis, Massachusetts Institute of Technology, May 1991. MIT AITR-1300.

[6] J. K. Salisbury, Jr., "Interpretation of contact geometries from force measurements," in Proceedings of IEEE International Conference on Robotics and Automation, pp. 240-247, March 1984.

[7] A. Bicchi, K. Salisbury, and D. Brock, "Contact sensing from force measurements," International Journal of Robotics Research, vol. 12, no. 3, pp. 249-262, 1993.

[8] Shadow Robot Company, "Shadow Dexterous Hand C5 technical specification." Published on www.shadowrobot.com, May 2008.
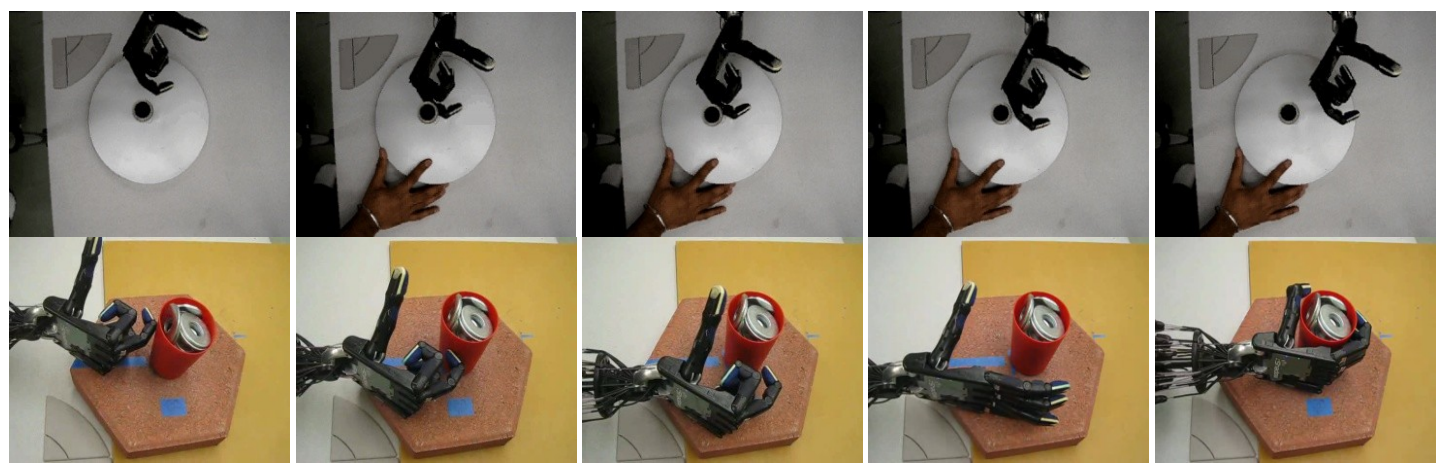

Fig 9. Top - Sequence showing obstacle avoidance, Bottom - Showing object localization and grasping 